\title{
THE CONTINUING CONCERN WITH TB
}

\section{TUBERCULOSIS (ICD-9 010-018)}

atest data on tuberculosis (Mycobacterium

tuberculosis, "TB") notifications for 1989 and 1990 show that TB continues to be a problem in NSW, and may be increasing. The rate of new cases of tuberculosis in NSW in 1989 was $4.89 / 100,000$, and 5.80/100,000 in 1990. The number of total mycobacteria notifications (including atypical mycobacteria) in 1990 was the highest for at least eight years (see Figure 3). The number of annual new TB cases over this period has not been determined; mainframe records containing these data will be analysed at a later stage.

There were 17 deaths from tuberculosis in 1989 (annual rate of $0.30 / 100,000$ population) and 20 deaths in 1990 (annual rate of $0.35 / 100,000$ ).

There were 14 reactivations of $\mathrm{M}$ tuberculosis in 1989 (4.5 per cent of notifications) and six reactivations in 1990 (1.7 per cent of notifications). In addition, there was one case reactivated atypical mycobacteria in each of the two years that we report.

There were 59 cases denotifiec in both 1989 and 1990 . This reflects the situation that tuberculosis notifactions are made provisionally, with microbiological confirmation coming up to two months later. Total notifications reflect the workload of the tuberculosis service with regard to contact tracing.

Case definitions used in this report are:

New TB:

- A patient who has a diagnosis of TB with culture confirmation of Mycobacterium tuberculosis, or A patient diagnosed as having active TB on clinical and/or radiological grounds, in the absence of bacteriological evidence, and who is receiving a course of anti-tuberculous chemotherapy.

$\mathrm{TB}$ reactivation:

- A patient diagnosed again as having active TB who has previously been notified in NSW and had received a recognised course of antituberculous chemotherapy. (Patients with a history of TB and/or treatment prior to coming to NSW are classified as new cases.)

\section{"Atypical" mycobacterial infection:}

- A patient with a clinically apparent infection caused by bacteriologically confirmed "atypical" mycobacteria, or

- A patient from whom bacteriologically confirmed "atypical" mycobacteria are recovered from sites which are normally sterile, or A patient from whom the same "atypical" mycobacterium has been isolated in moderate amounts from the same site.

The figures relate to new cases unless otherwise stated. Table 3 presents more details on the 1989 and 1990 TB data. The table shows that new TB cases made up fewer than 60 per cent of all mycobacteria notifications during these years. More than three-quarters of new TB cases were pulmonary in site. Total new TB cases rose 20 per cent from 1989 to 1990 , although this may be within normal year-to-year variability.

\section{TABLE 3}

MYCOBACTERIA NOTIFICATIONS

NSW, 1989 AND 1990

\begin{tabular}{|l|r|r|r|r|}
\hline \multirow{2}{*}{} & \multicolumn{2}{|c|}{1989} & \multicolumn{2}{c|}{1990} \\
\cline { 2 - 5 } & No. & $\%$ & No. & $\%$ \\
\hline Total mycobacteria notifications & 515 & 100 & 584 & 100 \\
Cases denotified & 59 & 11.5 & 59 & 10.1 \\
New TB cases - total & 310 & 60.2 & 346 & 59.2 \\
New TB cases - pulmonary & 241 & $(77.7)$ & 263 & $(76.0)$ \\
New TB cases - extrapulmonary & 69 & $(22.3)$ & 83 & $(23.9)$ \\
Atypical mycobacteria & 14.6 & 28.3 & 179 & 30.7 \\
\hline
\end{tabular}

Figure 4 shows new reported cases of tuberculosis for 1990 by age and sex. There is a male preponderance of cases (199 of $338-59$ per cent). The highest incidence occurred in the $20-39$ years and 60 years and over age groups. The 1989 data also demonstrate a male preponderance and peak incidences in these age groups. This is consistent with previous age distribution patterns for tuberculosis in NSW.

Rates for new cases by Area/Region in 1990 are shown in Figure 5. Note that the data for Wentworth and Western Sydney Areas have been combined to produce a single rate. The Areas predominate, particularly those of the Sydney metropolitan region, with the highest rate in the Central Sydney Area. This distribution may be explained by greater population densities and high numbers of overseas-born residents in these areas. It is of note that Central Sydney has an incidence of TB more than twice that of the overall NSW rate, and that all Sydney metropolitan Areas except Northern Sydney have incidence rates above the State average. However, these rates have not been adjusted for age or sex.

Table 4 provides a breakdown by region of birth/ Aboriginality. Of note is the very low rate among Australian Aborigines, and a high rate among Asianborn immigrants, reflecting the endemicity of TB in that region.

\begin{tabular}{|c|c|c|c|c|}
\hline \multicolumn{5}{|c|}{$\begin{array}{l}\text { NEW TB CASES BY } \\
\text { BIRTHPLACE AND ABORIGINALITY, } \\
\text { NSW, } 1989 \text { AND } 1990\end{array}$} \\
\hline & \multicolumn{2}{|c|}{1989} & \multicolumn{2}{|c|}{1990} \\
\hline & No. & $\%$ & No. & $\%$ \\
\hline Australia (caucasian) & 83 & 26.8 & 90 & 26.0 \\
\hline Australia (Aboriginal) & 4 & 1.3 & 3 & 0.9 \\
\hline Asia & 135 & 43.5 & 158 & 45.7 \\
\hline Europe & 34 & 11.0 & 38 & 11.0 \\
\hline Oceania & 11 & 3.5 & 16 & 4.6 \\
\hline Others & 43 & 13.9 & 41 & 11.8 \\
\hline
\end{tabular}

In 1990 there was no clear temporal or seasonal occurrence of new TB cases (Figure 6). Reports may be low in December due to low patient attendance or reporting activity in that month.

Overall, the data show that TB continues to be a problem in NSW, and may be increasing. Considerable variation exists in TB rates across Areas/Regions of the State, with the highest rates occurring in the Central, Eastern and Southern Sydney Areas.

Prevention of tuberculosis will continue to depend on early detection (including investigation of contacts and, where appropriate, screening of high-risk groups), effective treatment and adequate follow-up of both treated and suspected cases. 


\section{Improving AIDS surveillonce}

\section{FIGURE 3}

\section{MYCOBACTERIA NOTIFICATIONS}

NSW, 1982-1990

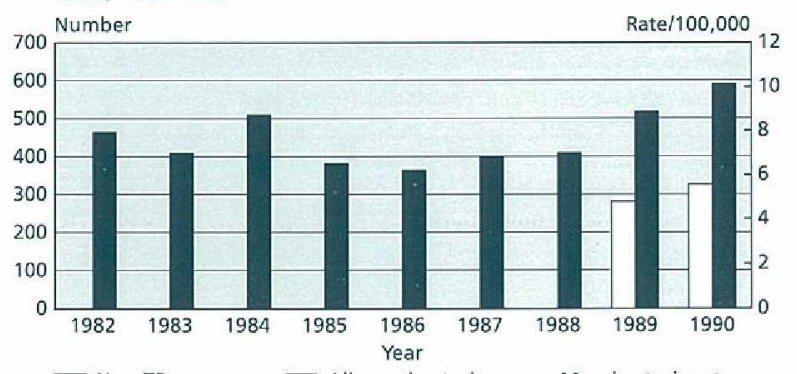

$\square$ New TB cases $\square$ All mycobacteria Mycobacteria rate

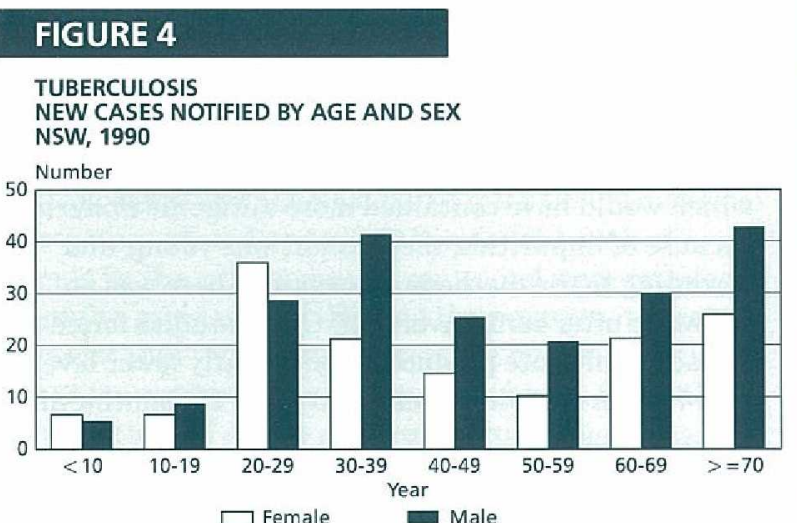

\section{FIGURE 5}

TUBERCULOSIS

NEW CASES NOTIFIED

BY AREA/REGION, NSW, 1990

Rate $/ 100,000$

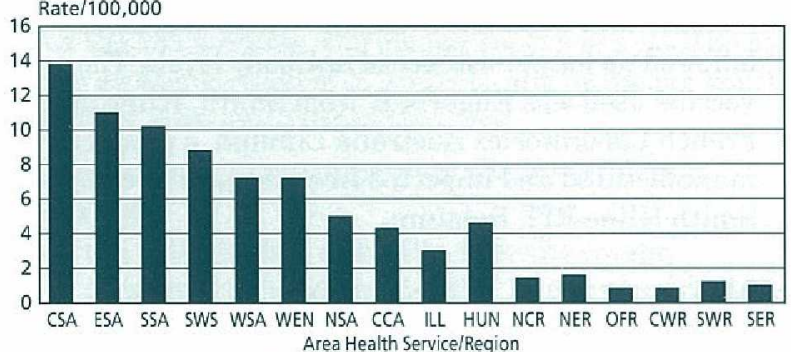

Note: horizontal line $=$ State incidence

\section{FIGURE 6}

\section{TUBERCULOSIS}

NEW CASES NOTIFIED

BY MONTH, NSW, 1990

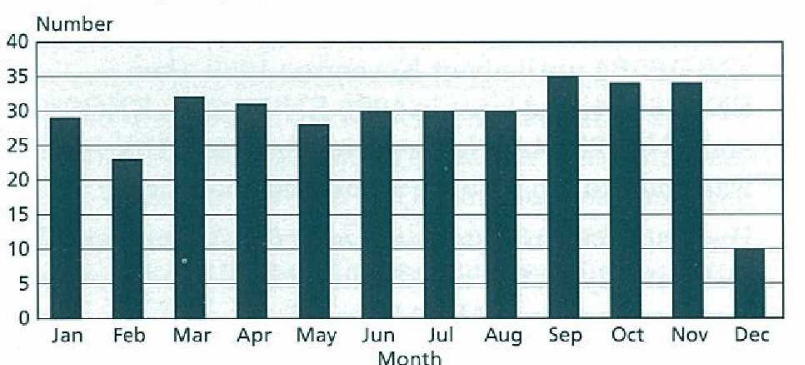

\section{Continued from page 100}

complex and at times vague and ambiguous. It also appears to be deficient; for example, HIV-related thrombocytopaenia, which may cause gastrointestinal haemorrhage and death, is not included in the definition as a specific entity. The case definition takes no account of immune system status, so a patient with a CD4 count of zero may never constitute a case. Additionally, prophylactic or other treatments may prevent or shorten or lessen the severity of attacks of illness so the threshold of AIDS diagnosis (for example, for PCP, 'chronic HSV' or oesophageal candidiasis) is never reached.

Funding formulas that rely on numbers of AIDS cases diagnosed, using the current definition, may therefore underestimate the resources required for dealing with HIV-related disease.

In conclusion, possible improvements to on-going AIDS surveillance noted during our review include the following:

1. Clinical services treating substantial numbers of HIVpositive people should maintain a computer database of clients, containing fields at least for seropositivity, CDC stage, significant diagnoses and whether notified, if a case.

2. A record should be kept in patients' files indicating whether they have been notified if they fulfil AIDSdefining criteria.

3. It would be valuable if one senior, motivated clinician were made responsible for correct and timely notification in all mutliple-doctor centres. They could provide copies of the most recent CDC/MMWR case definition for AIDS, as well as material on the importance of notification, to all doctors in contact with clients/patients in their centre. Regular reminders may also be necessary.

4. There should be feedback to doctors after notification - a receipt for the notification has been suggested and may be instituted. The circulation of a regularly updated (confidential) list of cases notified by a centre to its practising doctors may be sufficient.

5. Depending on the size and type of institution, a regular review could be undertaken of records of HIV positive patients to look for missed notifications.

6. Clear guidelines on who is responsible for notification also need to be provided to all centres. Perhaps both referring and tertiary treatment centres should be asked to notify, with duplication detected and removed by the Epidemiology Branch.

Many of these facilities or procedures already exist in some centres, or are being implemented.

\section{Mark Beh}

Public Health Officer, Epidemiology \& Health Services

Evaluation Branch, NSW Health Department

Virginia Furner

Medical Officer, Albion Street Clinic

Julian Gold

Director, Albion Street Clinic

1. Revision of the CDC Surveillance Case Definition for Acquired Immunodeficiency Syndrome, MMWR 1987;36(1S): (whole supplement). 2. Whyte BM, Swanson CE, Cooper DA. Survival of patients with the acquired immunodeficiency syndrome in Australia. Med J Aust acquired immunodef. 